\title{
Transmission Electron Microscopy Applications in the Semiconductor Industry - Challenges and Solutions for Specimen Preparation
}

\author{
Youren $\mathrm{Xu}$ and Chris Schwappach
}

Intel Corporation, New Mexico Materials Lab, 4100 Sara Road, Rio Rancho, NM 87124

Driven by Moore's law, transmission electron microscopy (TEM) has become an increasingly powerful technique supporting the development and manufacturing of deep sub-micron integrated circuits (ICs) due to its high resolution and nano-spot analysis capabilities [1]. The run rate of TEM analysis for major memory or logic chip manufacturers is about several thousand specimens per year. High precision and fast through-put time (TPT) are the two key factors that will determine if TEM can meet the growing demand for problem solving. The demand is driven by the increasing needs from new process development as well as from high volume manufacturing environments where a quick response to process excursion/line down situations is required. The introduction of the focused ion beam (FIB) technique and the emergence of the wedge (tripod polisher) method [2] achieved a breakthrough in TEM specimen preparation to meet these challenges. From a practical standpoint, overall specimen quality (thickness) and lack of end-point precision are two major issues associated with the conventional single beam FIB technique. These issues are primarily related to ion beam damage and endpoint control encountered during the final stages of specimen thinning.

The dual beam -focused ion beam (DB -FIB) system demonstrates superior performance over the single beam system including reduced TPT, ability to precisely section features in the sub micron range, high accuracy for endpoint control, and higher success rates. The DB-FIB technique can also be used to prepare high-precision plan-view TEM specimens [3]. Although the use of DB-FIB systems has greatly reduced specimen damage caused by the ion beam due to the added electron beam viewing capability, improper operating procedures can still induce unwanted ion beam damage. Fig. 1 shows a case where the Si substrate underneath the Ti/TiN adhesion layer was damaged due to missing e-beam Pt deposition prior to ion beam deposition. Our preliminary studies concluded that a minimum of 660 angstroms e-beam assisted Pt deposition is required for complete protection from a subsequent $350 \mathrm{pA}$ ion beam assisted Pt deposition (bulk layer).

The mechanical grinding/polishing operation is always one of the most critical steps for TEM specimen preparation. It is a slow and labor-intensive process. Sela's "TEMstation" (now called "TEMpro") system offers a solution to this problem. This newly emerged automatic TEM specimen prep system eliminates manual grinding/polishing for the entire FIB sliver preparation, which is normally performed by a highly skilled expert technician. Our evaluation data showed that the average TPT for sliver preparation is 18 minutes per specimen and the success rate is $\sim 90 \%$. The "TEMstation" can produce standard slivers ( $30 \mu \mathrm{m}$ thickness) from both inline and end of line wafers $(0.4 \mathrm{~mm}$ and $0.7 \mathrm{~mm}$ thickness $)$. The total sliver thickness $(\mathrm{Si}+$ grid $)$ is $\sim 200 \mu \mathrm{m}$ which fits both Philips and JEOL TEM specimen holders. The training needed for tool operation is less than a week for a person who does not have any grinding/polishing skills. This added capability will significantly improve the lab efficiency by better utilizing resources across functional areas.

The wedge method is a low cost approach offering a large transparent area with minimal or no ion milling. It is also a fast technique suitable for preparing specimens with repeatable structures. 
Currently, $30 \%$ of specimens in our lab are prepared using this method. However, extensive training and exercise is needed to become proficient with this technique. The nearly $100 \%$ manual preparation and personnel-dependent operation procedure seems to prevent the adoption of wedge as the primary technique by TEM labs as compared with the DB-FIB cross-section method which offers standard and easy procedures and the added benefit of SEM for monitoring the thinning process.

The FIB "lift-out" method has become increasingly attractive to the TEM community due to the advantage of no mechanical grinding/polishing [4]. This technique can be used to prepare multiple specimens from within a very small area offering a powerful solution for failure analysis where multi-site sampling is required due to imprecise fault isolation [5]. We have successfully prepared two samples that were $5.5 \mu \mathrm{m}$ apart from an FA unit using this technique (Fig. 2).

\section{References}

1. J Mardinly, Microscopy and Microanalysis, vol.7, supplement 2 (2001) 510.

2. S.J. Klepeis et al., Mater. Res. Soc. Symp. Proc., vol. 115 (1988) 179.

3. Z. Ma et al., Microscopy and Microanalysis, vol.5, supplement 2 (1999) 904.

4. L. Giannuzzi et al., Mat. Res. Soc. Symp. Proc., vol.480, (1997) 19.

5. Y. Xu, C. Schwappach, and R. Cervantes, Microscopy and Microanalysis, vol.6, supplement 2 (2000) 516.
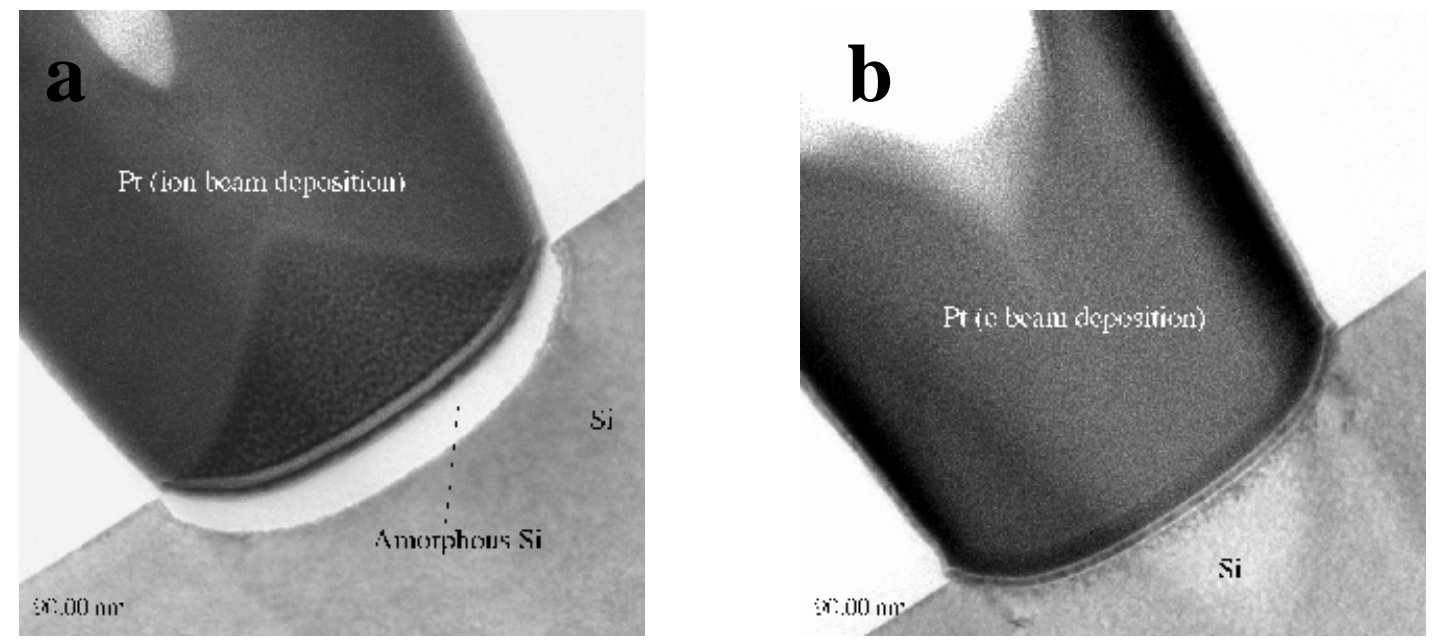

Figure 1. Use the proper Pt protective layer deposition: (a) ion beam Pt deposition caused Si amorphization; and (b) add a layer of e-beam deposited Pt prior to ion beam Pt deposition protecting the Si from ion damage.

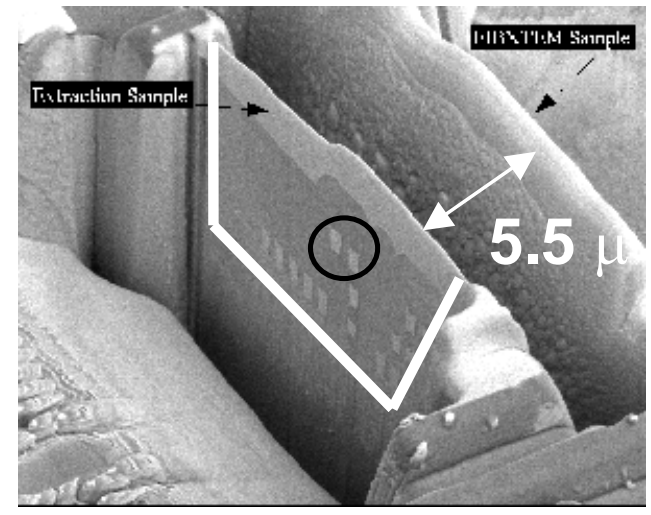

Figure 2. A case of multi-site sampling using the "liftout" method. The distance between the extracted sample and the conventional FIB cross-section sample is about 5.5 microns. 